\title{
Socio-Rational Secret Sharing as a New Direction in Rational Cryptography
}

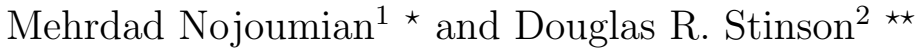 \\ 1 Department of Computer Science \\ Southern Illinois University, Carbondale, Illinois, USA \\ nojoumian@cs.siu.edu \\ 2 David R. Cheriton School of Computer Science \\ University of Waterloo, Waterloo, Ontario, Canada \\ dstinson@math. uwaterloo.ca
}

\begin{abstract}
Rational secret sharing was proposed by Halpern and Teague in [12]. The authors show that, in a setting with rational players, secret sharing and multiparty computation are only possible if the actual secret reconstruction round remains unknown to the players. All the subsequent works use a similar approach with different assumptions.

We change the direction by bridging cryptography, game theory, and reputation systems, and propose a "social model" for repeated rational secret sharing. We provide a novel scheme, named socio-rational secret sharing, in which players are invited to each game based on their reputations in the community. The players run secret sharing protocols while founding and sustaining a public trust network. As a result, new concepts such as a rational foresighted player, social game, and social Nash equilibrium are introduced.

To motivate our approach, consider a repeated secret sharing game such as "secure auctions", where the auctioneers receive sealed-bids from the bidders to compute the auction outcome without revealing the losing bids. If we assume each party has a reputation value, we can then penalize (or reward) the players who are selfish (or unselfish) from game to game. We show that this social reinforcement rationally stimulates the players to be cooperative.
\end{abstract}

Keywords: cryptography, game theory, reputation systems.

\section{Introduction}

The classical $(t, n)$-secret sharing scheme was proposed in $[29,5]$, where a dealer distributes shares of a secret $\alpha$ among $n$ players $P_{1}, \ldots, P_{n}$ for a subsequent secret recovery. In a Shamir secret sharing [29], the dealer first generates a random polynomial $f(x) \in \mathbb{Z}_{q}[x]$ of degree $t-1$ such that $f(0)=\alpha$ is the secret. He then sends shares $f(i)$ to player $P_{i}$ for $1 \leq i \leq n$. As a result, any group of $t$ or more

\footnotetext{
^ Research supported by NSERC CGS - MSFSS Supplements Program.

** Research supported by NSERC Discovery Grant 203114-12.
} 
players can reconstruct the secret by Lagrange interpolation whereas any group of size less than $t$ cannot gain any information about the secret. The standard assumption in traditional secret sharing is that each player is either honest (i.e., he follows protocols) or malicious (i.e., he deviates from protocols) where (1) at least $t$ honest parties cooperate in order to recover the secret, and (2) the total number of malicious players is less than $t$.

A new research direction was initiated by Halpern and Teague [12] in the area of secret sharing and multiparty computation in a game-theoretic setting. In this new scheme, players are rational rather than being honest or malicious. This means each player selects his action (i.e., revealing his share or not revealing it) based on the utility that he can gain. As illustrated by the authors, classical secret sharing fails in this setting due to the failure of the secret reconstruction round. We should highlight that, in the context of rational secret sharing, "deviation" means that a player has not revealed his share during the reconstruction phase. Sending incorrect shares is another issue which can be prevented by having the dealer sign the shares. For a simple example of such an authentication method, see [17]. We now provide a high-level description of the problem.

If players are primarily incentivized to learn the secret, and secondly, they prefer that fewer of the other parties learn it, then it is not reasonable for each player to reveal his share in the "recovery phase". For instance, suppose players $P_{1}, P_{2}, P_{3}$ receive shares $6,11,18$ from a dealer respectively, where $f(x)=3+$ $2 x+x^{2} \in \mathbb{Z}_{19}[x]$ is the secret sharing polynomial. If only two players reveal their shares in the recovery phase, then the third selfish player (who has not revealed his share) can reconstruct the secret using two revealed shares and his own private share. Obviously, the other two cooperative players who have revealed their shares do not learn the secret. This justifies why the players do not reveal their shares in a rational setting, i.e., each player waits to receive shares of the other parties (see $[7,15]$ for an overview in this direction).

To generalize this, consider the following scenario for a player $P_{j}$ where the degree of the secret sharing polynomial is $t-1$. If players $P_{i}$ (for $i$ less than $t-1$ or $i$ more than $t-1$ ) reveal their shares, nothing changes whether $P_{j}$ reveals his share or not. In the former case, no one learns the secret. In the latter case, everyone learns the secret. On the other hand, if exactly $t-1$ players $P_{i}$ reveal their shares, then $P_{j}$ can not only learn the secret with his own private share (i.e., $t$ shares are sufficient to use Lagrange interpolation) but also can prevent the other players from learning the secret by not revealing his share, i.e., achieving the second preference of a self-interested player in rational secret sharing. In other words, for each $P_{i}$, revealing the share is weakly dominated by not revealing the share. As a result, no one reveals his share and the secret is never reconstructed.

Before providing our solution to the rational secret sharing problem, we briefly introduce the notion of social secret sharing $[25,26]$ in which players are either honest or malicious. In this protocol, weights of the players, i.e., the number of shares each player can hold, are periodically updated such that the players who cooperate receive more shares than those who defect. Although this 
scheme addresses a different issue compared to the secret recovery problem in a rational setting, we use its trust function in order to construct a new solution concept in rational cryptography.

\subsection{Our Solution in Nutshell}

In our "socio-rational" setting, the players are "selfish" similar to standard rational secret sharing. In addition, they have "concerns" about future gain or loss since our secret sharing game is repeated an unknown number of times. We term this new type of the player, a rational foresighted player. In the proposed scheme, each player has a reputation value which is updated according to his behavior each time the game is played. The initial reputation value is zero and its computation is public. For instance, if a player cooperates (he reveals his share), his trust value is increased, otherwise, it is decreased. A long-term utility (used by each player for action selection) and an actual utility (used for the real payment at the end of each game) are computed based on the following parameters:

1. Estimation of future gain or loss due to trust adjustment (virtual utility).

2. Learning the secret at the current time (real utility).

3. The number of other players learning the secret at the moment (real utility).

All these factors are used by each player to estimate his long-term utility and consequently to select his action, whereas only the last two items are used to compute the real payment at the end of each game. To estimate future impact, the following scenario is considered: whenever a player cooperates (or defects), we assume he can potentially gain (or lose) some extra units of utility, i.e., he has a greater (or lesser) chance to be "invited" to the future games and consequently he gains (or loses) more utilities. In other words, if the reputation of $P_{i}$ is decreased, he will have less chance to be invited to the future secret sharing games. Otherwise, $P_{i}$ is going to be invited to more secret sharing games. To realize this scenario, in each game, the dealer selects the players based on their reputations, for instance, $50 \%$ from reputable players, $30 \%$ from newcomers, and $20 \%$ from non-reputable parties, where the number of players in each category possibly varies.

This gain or loss is "virtual" at the current time but will be "realized" in the future. As an example of "future impact", consider the following statements, where $\mathrm{U} \gg \mathrm{u}$ and $\mathrm{V} \gg \mathrm{v}$ :

1. As a consumer, if you buy something today (cooperate and lose $\$ \mathrm{u}$ ), you will receive a significant discount from the producer (rewarded $\$ \mathrm{U}$ ) on your next purchase.

2. As a producer, if you use low-grade materials to save some money (defect and gain $\$ \mathrm{v}$ ), you will lose many of your consumers (penalized $\$ \mathrm{~V}$ ) in the coming years.

In other words, if we construct a socio-rational model where the players can gain (or lose) more utility in the future games than the current game, depending on their behavior, we can then incentivize them to be foresighted and cooperative. 


\subsection{Our Motivation}

In secure multiparty computation $[9,4,6]$, various players cooperate to jointly compute a function based on the private data they each provide. As stated in the literature, secret sharing is a fundamental method that is used by the players to inject their private data into a multiparty computation protocol. At the end of a multiparty computation protocol, each player has a share of the function value. Therefore, they can collaborate to reveal this value to everyone.

We refer to sealed-bid auctions [13] as an application of multiparty computation. In a secure auction, auctioneers receive many sealed-bids from bidders and the goal is to compute the auction outcome (i.e., the winner and selling price) without revealing the losing bids. The main reason for using sealed-bids is the fact that, if bids are not kept private, they can be used in the future auctions and negotiations by different parties, say auctioneers, to maximize their revenues, or competitors, to win a later auction. To motivate our concept of "socio-rational secret sharing", consider the following repeated game, as shown in Figure 1:

1. The bidders select a subset of auctioneers based on a non-uniform probability distribution over the auctioneers' types, i.e., reputable auctioneers have a greater chance to be selected.

2. Each bidder then acts as an independent dealer to distribute the shares of his sealed-bid among the selected auctioneers.

3. Subsequently, the auctioneers compute the selling price and determine the winner by using a multiparty computation protocol.

4. In the last phase of the multiparty computation, the auctioneers reconstruct the selling price $\alpha$ and report it to the seller.

In this setting, only the auctioneers who have learned and reported $\alpha$ to the seller, are each paid $\$ \Omega$, i.e., there exists a "competition" for learning the secret. In addition, $\$ \Omega$ are divided among the auctioneers who have learned the secret; each of them can therefore earn more money if fewer of them learn $\alpha$. If we repeat this game an unknown number of times and choose an appropriate invitation mechanism based on the players' reputation, we can incentivize the auctioneers to be cooperative, that is, they will reveal the shares of $\alpha$ in the recovery phase.

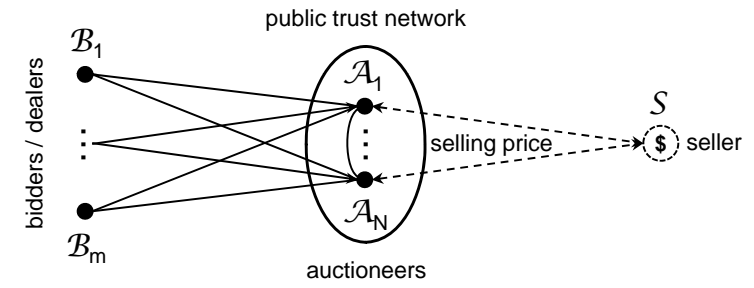

Fig. 1. Sealed-Bid Auction as a Repeated Secret Sharing Game 


\subsection{Our Contribution}

We provide a new solution concept to the rational secret sharing problem by considering a social setting in which the players enter into a long-term interaction for executing an unknown number of independent secret sharing protocols.

In our model, a public trust network is constructed in order to incentivize the players to be cooperative. This incentive is sustained from game to game since the players are motivated to enhance their reputations and consequently gain extra utility. In other words, they avoid a selfish behavior due to the social reinforcement of the trust network. Constructing a "social model" and inviting the players to a repeated game based on their "reputations" in the community, is a new contribution not only in rational cryptography but also in the existing game-theoretic solution concepts. We refer the reader to [21] for other discussions in this direction. Our scheme has the following desirable properties:

- It has a single secret recovery round, despite the existing solutions.

- It provides a game-theoretic solution that is always a Nash equilibrium.

- It is immune to rushing attack; it is not advantageous for players to wait.

- It prevents players from aborting the game; the case in some solutions.

The rest of this paper is organized as follows. Section 2 provides the relevant background. Section 3 reviews the literature of rational cryptography. Section 4 present our construction. Section 5 compares our solution with the existing schemes and techniques. Finally, Section 6 provides concluding remarks.

\section{Preliminaries}

In this section, some preliminaries regarding game-theoretic concepts along with social and rational secret sharing schemes are presented.

\subsection{Game-Theoretic Concepts}

A game consists of a set of players, a set of actions and strategies (i.e., the way of choosing actions), and finally a pay-off function which is used by each participant to compute his utility. In cooperative games, players collaborate and split the total utility among themselves, i.e., cooperation is enforced by agreements. In non-cooperative games, players can not form agreements to coordinate their behavior, i.e., any cooperation must be self-enforcing.

The prisoner's dilemma, shown in Figure 2, is an example of non-cooperative games. In this game, we have two possible actions: $\mathcal{C}$ : keep quiet (or cooperation) and $\mathcal{D}$ : confess (or defection). In the pay-off matrix, $+1,0,-1$, and -2 denote freedom, jail for one year, jail for two years, and jail for three years respectively. The outcome of this game is going to be $(\mathcal{D}, \mathcal{D})$ due to the Nash equilibrium concept, while the ideal outcome is $(\mathcal{C}, \mathcal{C})$. To analyze why the game has such an outcome, consider the following two scenarios: 
1. If $P_{1}$ selects $\mathcal{C}$ (the first row), then $P_{2}$ will select $\mathcal{D}$ (the second column) since $+1>0$.

2. If $P_{1}$ selects $\mathcal{D}$ (the second row), then $P_{2}$ will select $\mathcal{D}$ (the second column) since $-1>-2$.

This means that, regardless of whether $P_{1}$ cooperates or defects, player $P_{2}$ will always defect. Since the pay-off matrix is symmetric, we also have that, regardless of whether $P_{2}$ cooperates or defects, player $P_{1}$ will always defect. In other words, since players are in two different locations and cannot coordinate their behavior, the final outcome is going to be $(\mathcal{D}, \mathcal{D})$.

$P_{1}$

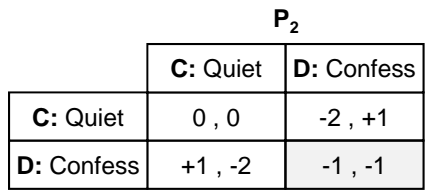

$P_{1}$

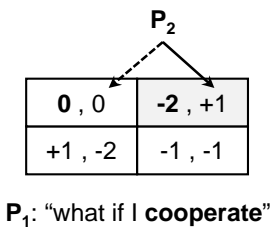

$\mathbf{P}_{\mathbf{1}}$ : "what if I defect"

$P_{1}$

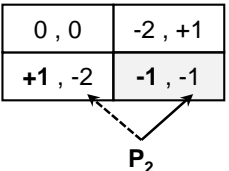

Fig. 2. Nash Equilibrium in Prisoner's Dilemma

Definition 1. Let $\mathcal{A} \stackrel{\text { def }}{=} \mathcal{A}_{1} \times \cdots \times \mathcal{A}_{n}$ be an action profile for $n$ players, where $\mathcal{A}_{i}$ denotes the set of possible actions of player $P_{i}$. A game $\Gamma=\left(\mathcal{A}_{i}, u_{i}\right)$ for $1 \leq i \leq n$, consists of $\mathcal{A}_{i}$ and a utility function $u_{i}: \mathcal{A} \mapsto \mathbb{R}$ for each player $P_{i}$. We refer to a vector of actions $\boldsymbol{a}=\left(a_{1}, \ldots, a_{n}\right) \in \mathcal{A}$ as an outcome of the game.

Definition 2. The utility function $u_{i}$ illustrates the preferences of player $P_{i}$ over different outcomes. We say $P_{i}$ prefers outcome $\boldsymbol{a}$ to $\boldsymbol{a}^{\prime}$ iff $u_{i}(\boldsymbol{a})>u_{i}\left(\boldsymbol{a}^{\prime}\right)$, and he weakly prefers outcome $\boldsymbol{a}$ to $\boldsymbol{a}^{\prime}$ if $u_{i}(\boldsymbol{a}) \geq u_{i}\left(\boldsymbol{a}^{\prime}\right)$.

In order to allow the players to follow randomized strategies (where the strategy is the way of choosing actions), we define $\sigma_{i}$ as a probability distribution over $\mathcal{A}_{i}$ for a player $P_{i}$. This means that he samples $a_{i} \in \mathcal{A}_{i}$ according to $\sigma_{i}$. A strategy is said to be a pure-strategy if each $\sigma_{i}$ assigns probability 1 to a certain action, otherwise, it is said to be a mixed-strategy. Let $\boldsymbol{\sigma}=\left(\sigma_{1}, \ldots, \sigma_{n}\right)$ be the vector of players' strategies, and let $\left(\sigma_{i}^{\prime}, \boldsymbol{\sigma}_{-i}\right) \stackrel{\text { def }}{=}\left(\sigma_{1}, \ldots, \sigma_{i-1}, \sigma_{i}^{\prime}, \sigma_{i+1}, \ldots, \sigma_{n}\right)$, where $P_{i}$ replaces $\sigma_{i}$ by $\sigma_{i}^{\prime}$ and all the other players' strategies remain unchanged. Therefore, $u_{i}(\boldsymbol{\sigma})$ denotes the expected utility of $P_{i}$ under the strategy vector $\boldsymbol{\sigma}$. A player's goal is to maximize $u_{i}(\boldsymbol{\sigma})$. In the following definitions, one can substitute an action $a_{i} \in \mathcal{A}_{i}$ with its probability distribution $\sigma_{i} \in \mathcal{S}_{i}$ or vice versa.

Definition 3. A vector of strategies $\boldsymbol{\sigma}$ is a Nash equilibrium if, for all $i$ and any $\sigma_{i}^{\prime} \neq \sigma_{i}$, it holds that $u_{i}\left(\sigma_{i}^{\prime}, \boldsymbol{\sigma}_{-i}\right) \leq u_{i}(\boldsymbol{\sigma})$. This means no one gains any advantage by deviating from the protocol as long as the others follow the protocol. 
Definition 4. Let $\mathcal{S}_{-i} \stackrel{\text { def }}{=} \mathcal{S}_{1} \times \cdots \times \mathcal{S}_{i-1} \times \mathcal{S}_{i+1} \times \cdots \times \mathcal{S}_{n}$. A strategy $\sigma_{i} \in \mathcal{S}_{i}$ (or an action) is weakly dominated by a strategy $\sigma_{i}^{\prime} \in \mathcal{S}_{i}$ (or another action) with respect to $\mathcal{S}_{-i}$ if:

1. For all $\boldsymbol{\sigma}_{-i} \in \mathcal{S}_{-i}$, it holds that $u_{i}\left(\sigma_{i}, \boldsymbol{\sigma}_{-i}\right) \leq u_{i}\left(\sigma_{i}^{\prime}, \boldsymbol{\sigma}_{-i}\right)$.

2. There exists a $\boldsymbol{\sigma}_{-i} \in \mathcal{S}_{-i}$ such that $u_{i}\left(\sigma_{i}, \boldsymbol{\sigma}_{-i}\right)<u_{i}\left(\sigma_{i}^{\prime}, \boldsymbol{\sigma}_{-i}\right)$.

This means that $P_{i}$ can never improve its utility by playing $\sigma_{i}$, and he can sometimes improve it by not playing $\sigma_{i}$. A strategy $\sigma_{i} \in \mathcal{S}_{i}$ is strictly dominated if player $P_{i}$ can always improve its utility by not playing $\sigma_{i}$.

\subsection{Rational Secret Sharing}

In this section, we review rational secret sharing, which was initiated by Halpern and Teague [12]. The scheme consists of a dealer $D$, who creates a secret sharing scheme with threshold $t$, and $n$ players $P_{1}, \ldots, P_{n}$.

The protocol proceeds in a sequence of iterations where only one iteration is the "real" secret recovery phase (i.e., the last iteration) and the rest are just "fake" iterations for trapping selfish players. At the end of each iteration, the protocol either terminates (due to the observation of selfish behavior or cooperation for secret recovery) or it proceeds to the next iteration. Indeed, in any given round, players do not know whether the current iteration is the real recovery phase (where a player may gain more utility by being silent and not sending his share to others), or just a test round. The following steps (a)-(d) provide a description of the initial solution to a rational secret sharing game, where $n=3, t=3$, and shares are revealed simultaneously, as shown in $[12,10]$. Table 1 shows all the different possibilities that can occur.

(a) In each round, $D$ initiates a fresh secret sharing scheme where each player $P_{i}$ receives a share $f_{i}$.

(b) During an iteration, each player $P_{i}$ flips a biased coin $c_{i} \in\{0,1\}$ where $\operatorname{Pr}\left[c_{i}=1\right]=\rho$.

(c) Players compute $c^{*}=\oplus c_{i}$ by a multiparty computation protocol without revealing $c_{i}$-s.

(d) Now $c^{*}$ is known to everyone. If $c^{*}=c_{i}=1, P_{i}$ broadcasts his share. We then have:

(d.1) If three shares are revealed, the secret is recovered and the protocol ends.

(d.2) If $c^{*}=1$, and no share or two shares are revealed, players terminate the protocol.

(d.3) In any other cases, the dealer and players proceed to the next round, i.e., step (a).

To see how the above protocol works, assume $P_{1}, P_{2}$ follow the protocol whereas $P_{3}$ is willing to deviate. He may deviate in "coin-tossing" or in "revealing" his share. We should note that each $P_{i}$ selects $c_{i}$ independently. The following cases are different possible deviation scenarios: 


\begin{tabular}{|c|c|c|c|c|c|}
\hline Rows & $c_{1}$ & $c_{2}$ & $c_{3}$ & Public $c^{*}$ & Revealed Shares \\
\hline \hline 1 & 0 & 0 & 0 & 0 & - \\
\hline 2 & 0 & 0 & 1 & 1 & $f_{3}$ \\
\hline 3 & 0 & 1 & 0 & 1 & $f_{2}$ \\
\hline 4 & 0 & 1 & 1 & 0 & - \\
\hline 5 & 1 & 0 & 0 & 1 & $f_{1}$ \\
\hline 6 & 1 & 0 & 1 & 0 & - \\
\hline 7 & 1 & 1 & 0 & 0 & - \\
\hline 8 & 1 & 1 & 1 & 1 & $f_{1}, f_{2}, f_{3}$ \\
\hline
\end{tabular}

Table 1. Three-Player Rational Secret Sharing Game

- It is not advantageous for $P_{3}$ to bias $c_{3}$ to be 0 with higher probability, since, when $c_{3}=0$, either no share or one share is revealed.

- It is also not advantageous for $P_{3}$ to bias $c_{3}$ to be 1 with higher probability, since, when $c_{3}=1$, either no share, or one share, or all shares are revealed. This may lead to an "early" secret recovery but it does not have any effect of the utility of $P_{3}$.

- If $c_{3}=0$ or $c^{*}=0$ (that is, one of rows $1,3,4,5,6,7$ in Table 1 occurs), then there is no incentive for $P_{3}$ to deviate since in all these cases he is supposed not to reveal his share.

- If $c_{3}=1$ and $c^{*}=1$ (that is, one of rows 2,8 in Table 1 occurs), then player $P_{3}$ is supposed to reveal his share. There exist two possibilities:

1. $c_{1}=1$ and $c_{2}=1$, which occurs with the following probability:

$$
\begin{aligned}
\operatorname{Pr}\left[c_{1}=1 \wedge c_{2}=1 \mid c_{3}=1 \wedge c^{*}=1\right] & =\frac{\operatorname{Pr}\left[c_{1}=1 \wedge c_{2}=1 \wedge c_{3}=1\right]}{\operatorname{Pr}\left[c_{3}=1 \wedge c^{*}=1\right]} \\
& =\frac{\rho^{3}}{(1-\rho)(1-\rho) \rho+\rho^{3}} \\
& =\frac{\rho^{2}}{(1-\rho)^{2}+\rho^{2}} .
\end{aligned}
$$

2. $c_{1}=0$ and $c_{2}=0$, which occurs with the remaining probability:

$$
\begin{aligned}
\operatorname{Pr}\left[c_{1}=0 \wedge c_{2}=0 \mid c_{3}=1 \wedge c^{*}=1\right] & =\frac{\operatorname{Pr}\left[c_{1}=0 \wedge c_{2}=0 \wedge c_{3}=1\right]}{\operatorname{Pr}\left[c_{3}=1 \wedge c^{*}=1\right]} \\
& =\frac{(1-\rho)(1-\rho) \rho}{(1-\rho)(1-\rho) \rho+\rho^{3}} \\
& =\frac{(1-\rho)^{2}}{(1-\rho)^{2}+\rho^{2}} .
\end{aligned}
$$


Therefore, if player $P_{3}$ deviates by not revealing his share, either he is going to be the only player who learns the secret or the protocol terminates and he never learns the secret. Let assume player $P_{3}$ gains $U^{+}$if he is the only player who learns the secret, let $U$ denotes the utility gain for each $P_{i}$ if all three players learn the secret, and let $U^{-}$denotes the utility gain, say $\$ 0$, for each player $P_{i}$ if no one learns the secret. It is assumed that $U^{+}>U>U^{-}$. Therefore, a rational $P_{3}$ will cheat only if:

$$
U^{+}\left(\frac{\rho^{2}}{(1-\rho)^{2}+\rho^{2}}\right)+U^{-}\left(\frac{(1-\rho)^{2}}{(1-\rho)^{2}+\rho^{2}}\right)>U .
$$

If we assign an appropriate value to $\rho$, based on the players' utility function, such that the inequality (1) is not satisfied, then $P_{3}$ has no incentive to deviate when $c_{3}=1$ and $c^{*}=1$.

The authors in [12] also showed that this three-player game can be generalized to a game with $n$ players. As we just stated, certain assumptions regarding the players' utility function are required for rational secret sharing to be achievable. Let $u_{i}(\boldsymbol{a})$ denotes the utility of $P_{i}$ in a specific outcome $\boldsymbol{a}$ of the protocol. Suppose $l_{i}(\boldsymbol{a})$ is a bit defining whether $P_{i}$ has learned the secret or not in $\boldsymbol{a}$. We then define $\delta(\boldsymbol{a})=\sum_{i} l_{i}(\boldsymbol{a})$, which denotes the number of players who have learned the secret. The generalized assumptions of rational secret sharing are as follows:

- $l_{i}(\boldsymbol{a})>l_{i}\left(\boldsymbol{a}^{\prime}\right) \Rightarrow u_{i}(\boldsymbol{a})>u_{i}\left(\boldsymbol{a}^{\prime}\right)$.

- $l_{i}(\boldsymbol{a})=l_{i}\left(\boldsymbol{a}^{\prime}\right)$ and $\delta(\boldsymbol{a})<\delta\left(\boldsymbol{a}^{\prime}\right) \Rightarrow u_{i}(\boldsymbol{a})>u_{i}\left(\boldsymbol{a}^{\prime}\right)$.

The first assumption means $P_{i}$ prefers an outcome in which he learns the secret, that is, since $l_{i}(\boldsymbol{a})=1$ and $l_{i}\left(\boldsymbol{a}^{\prime}\right)=0$, he therefore prefers $\boldsymbol{a}$. The second assumption means $P_{i}$ prefers an outcome in which the fewest number of other players learn the secret, given that $P_{i}$ learns (or does not learn) the secret in both outcomes.

\subsection{Social Secret Sharing}

Now we review social secret sharing, introduced by Nojoumian et al. [25], where the shares are allocated based on a player's reputation and the way she interacts with other parties. In other words, weights of players are adjusted such that participants who cooperate receive more shares compared to non-cooperative parties. This is similar to human social life where people share more secrets with whom they really trust and vice versa. In the context of social secret sharing, the players are either honest or malicious.

To quantify the reputation of each player in a social secret sharing scheme, we apply the trust calculation method proposed in [24] (which is the modified version of the solution in [30]). We start with the following definition:

Definition 5. Let $\mathcal{T}_{i}^{j}(p)$ be the trust value assigned by $P_{j}$ to $P_{i}$ in time interval p. $\mathcal{T}_{i}: \mathbb{N} \mapsto \mathbb{R}$ is the trust function used to compute the reputation of $P_{i}$ : 


$$
\mathcal{T}_{i}(p)=\frac{1}{n-1} \sum_{j \neq i} \mathcal{T}_{i}^{j}(p),
$$

where $-1 \leq \mathcal{T}_{i}(p) \leq+1$ and $\mathcal{T}_{i}(0)=0$, i.e., we calculate the average of $n-1$ trust values (personal quantities) to compute a player's reputation (a social quantity).

For instance, let the trust values of $P_{1}, P_{2}$, and $P_{3}$ with respect to $P_{4}$ be $\mathcal{T}_{4}^{1}(p)=0.4, \mathcal{T}_{4}^{2}(p)=0.5$, and $\mathcal{T}_{4}^{3}(p)=0.6$. As a result, reputation of $P_{4}$ will be $\mathcal{T}_{4}(p)=0.5$. In this paper, only a public value $\mathcal{T}_{i}(p)$ is assigned to each player $P_{i}$, where $\mathcal{T}_{i}(p)$ represents his reputation. Equivalently, we can assume that $\mathcal{T}_{i}(p)=\mathcal{T}_{i}^{j}(p)$ for all $j$.

We now briefly review the approach proposed in [24]. As shown in Table 2, three "types" of players (that is, $\mathcal{B}$ : bad; $\mathcal{N}$ : new; and $\mathcal{G}$ : good) with six possible outcomes are defined, where $\alpha$ and $\beta$ determine boundaries on the trust values used to define the different sets of players. This approach then applies functions $\mu(x)$ and $\mu^{\prime}(x)$ respectively to update the reputation of each player $P_{i}$, as shown in Figure 3. Parameters $\eta, \theta$, and $\kappa$ are used to increment and/or decrement the trust value of a player. In intervals $[1-\epsilon,+1]$ and $[-1, \epsilon-1]$, functions $\mu(x)$ and $\mu^{\prime}(x)$ both converge to 0 , as required by Definition 5 .

\begin{tabular}{|ccc|c|c|}
\hline \multicolumn{2}{|c|}{ Current } & Trust Value & Cooperation & Defection \\
\hline \hline$P_{i} \in \mathcal{B}$ & if & $\mathcal{T}_{i}(p) \in[-1, \beta)$ & Encourage & Penalize \\
\hline$P_{i} \in \mathcal{N}$ & if & $\mathcal{T}_{i}(p) \in[\beta, \alpha]$ & Give a Chance & Take a Chance \\
\hline$P_{i} \in \mathcal{G}$ & if & $\mathcal{T}_{i}(p) \in(\alpha,+1]$ & Reward & Discourage \\
\hline
\end{tabular}

Table 2. Six Possible Actions for the Trust Management
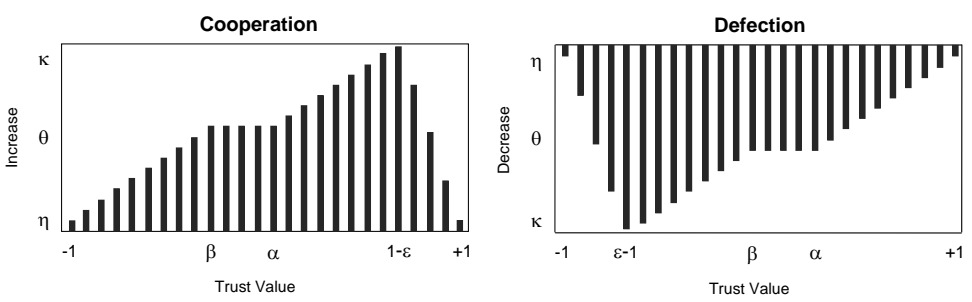

Fig. 3. Trust Adjustment by $\mu(x)$ and $\mu^{\prime}(x)$ Functions

We should stress that our trust function is not just a function of a single round, but of the "players' history". That is, it rewards more the better a participant has been, e.g., see Figure 3: Cooperation, where $\mathcal{T}_{i}(p) \in[\alpha, 1-\epsilon]$, and it penalizes more the worse a participant has been, e.g., see Figure 3: Defection, where $\mathcal{T}_{i}(p) \in[\epsilon-1, \beta]$. In addition, it provides opportunities for newcomers to 
increase their trust values even where we do not know much about their behavior, e.g., see Figure 3: where $\mathcal{T}_{i}(p) \in[\beta, \alpha]$.

Let $\ell_{i} \in\{0,1\}$ where $\ell_{i}=1$ denotes that player $P_{i}$ has cooperated in the current period and $\ell_{i}=0$ denotes that he has defected. The proposed trust function is as follows, where $x=\mathcal{T}_{i}(p-1)$ (i.e., $x$ is the previous trust value):

$$
\begin{aligned}
& \ell_{i}=1 \quad \Rightarrow \quad \mathcal{T}_{i}(p)=\mathcal{T}_{i}(p-1)+\mu(x) \text {, where } \\
& \mu(x)= \begin{cases}\frac{\theta-\eta}{\beta+1}(x+1)+\eta & P_{i} \in \mathcal{B} \\
\theta & P_{i} \in \mathcal{N} \\
\frac{\kappa-\theta}{1-\epsilon-\alpha}(x-\alpha)+\theta & P_{i} \in \mathcal{G}, \mathcal{T}_{i}(p) \leq 1-\epsilon \\
\frac{\kappa}{\epsilon}(1-x-\epsilon)+\kappa & \mathcal{T}_{i}(p)>1-\epsilon\end{cases} \\
& \ell_{i}=0 \quad \Rightarrow \quad \mathcal{T}_{i}(p)=\mathcal{T}_{i}(p-1)-\mu^{\prime}(x), \text { where } \\
& \mu^{\prime}(x)= \begin{cases}\frac{\kappa}{\epsilon}(x+1) & \mathcal{T}_{i}(p)<\epsilon-1 \\
\frac{\theta-\kappa}{\beta-\epsilon+1}(x-\epsilon+1)+\kappa & P_{i} \in \mathcal{B}, \mathcal{T}_{i}(p) \geq \epsilon-1 \\
\theta & P_{i} \in \mathcal{N} \\
\frac{\eta-\theta}{1-\alpha}(x-\alpha)+\theta & P_{i} \in \mathcal{G}\end{cases}
\end{aligned}
$$

Each function $\mu(x)$ and $\mu^{\prime}(x)$ consists of four linear equations, each of which is simply determined by two points $\left(x_{1}, y_{1}\right)$ and $\left(x_{2}, y_{2}\right)$ as follows:

$$
y=\frac{y_{2}-y_{1}}{x_{2}-x_{1}}\left(x-x_{1}\right)+y_{1} .
$$

To ensure that $\mathcal{T}_{i}(p-1)+\mu(x) \leq 1$ and $\mathcal{T}_{i}(p-1)-\mu^{\prime}(x) \geq-1$ when $x=1-\epsilon$ and $x=\epsilon-1$ respectively, we must satisfy the conditions $1-\epsilon+\kappa \leq 1$ and $\epsilon-1-\kappa \geq-1$, or equivalently $\kappa \leq \epsilon$. This is sufficient to ensure that $\mathcal{T}_{i}(p)$ never exceeds +1 or -1 .

It is worth mentioning that the authors in [24] also define an additional parameter as the transaction cost to deal with cheap cooperations and expensive defections. For instance, consider a scenario in which a player cooperates in regular transactions for several times in order to gain a high trust value. He can then defect in a critical transaction to severely damage the scheme. By considering this transaction cost parameter, a weight for "cooperation" or "defection" is defined and accordingly the trust value is adjusted.

\section{Literature Review}

As we mentioned, the notion of rational secret sharing was introduced by Halpern and Teague [12]. Assuming the same game-theoretic model, Lysyanskaya and 
Triandopoulos [20] provide a solutions in a mixed-behavior setting in which players are either rational or malicious. Abraham et al. [1] define a notion of resistance to coalitions and present a coalition-resistant protocol. All these constructions use simultaneous channels (either a broadcast channel or secure private channels) that means each player must decide on the value he wants to broadcast before observing the values broadcasted by the other players; this is known as a strategic game.

The proposed protocols in $[18,19,14]$ rely on physical assumptions such as secure envelopes and ballot boxes, which might be impossible or difficult to implement. In the same model, Micali and Shelat [23] provided a purely rational secret sharing scheme using a verifiable trusted channel. They showed that all the existing solutions not only rely on the players' rationality, but also on their beliefs. As a result, they cannot guarantee that all rational players learn the secret. For instance, suppose $P_{i}$ believes that equilibrium $(a, b)$ is played whereas $P_{j}$ believes $\left(a^{\prime}, b^{\prime}\right)$ is played, but the game leads to $\left(a, b^{\prime}\right)$, which may not be an equilibrium at all.

Kol and Naor [17] introduced an equilibrium notion, termed strict Nash equilibrium, in an information-theoretic secure setting. In a Nash equilibrium, no deviations are advantageous (i.e., there is no incentive to deviate). In its strict counterpart, all deviations are disadvantageous (i.e., there is an incentive not to deviate). They first considered both simultaneous and non-simultaneous broadcast channels and provided a new solution to avoid the simultaneous channel at the cost of increasing the round complexity.

Kol and Naor later [16] showed that all the existing computational-based protocols are susceptible to backward induction because of the cryptographic primitives used in the beginning of those protocols. That is, they can surely be broken after an exponential number of rounds. The authors then illustrate a new cryptographic coalition-resilient approach that is immune to backward induction by considering simultaneous as well as non-simultaneous broadcast channels.

The notion of computational strict Nash equilibrium was introduced in [8]. This construction is dealer-free and can tolerate a coalition of size $t-1$ without using simultaneous channels. It can even be run over asynchronous point-topoint networks. Finally, it is efficient in terms of computation, share size, and round complexity.

Maleka et al. [22] presented repeated rational secret sharing, with the same approach proposed in [28], by considering two punishment strategies. In the former, each player reveals his share as long as the other players cooperate. As soon as the first defection is observed, the players do not reveal their shares in every subsequent game. In the latter, the players do not send their shares to the deviant for $k$ subsequent games after observing the first defection. In the first scheme, each player not only punishes the deviant but also the other players including himself. In the second method, a player may deviate in an expensive secret recovery without having any concern for $k$ subsequent cheap reconstructions. Indeed, the nature of a punishment strategy must depend on how much 
future outcomes are worth for each player. Finally, they only considered a fixed number of $m$ players without allowing newcomers to join the scheme.

Other results have recently been proposed in the literature. For instance, Ong et al. [27] presented a protocol that is fair when the reconstruction phase is executed with many rational players together with a minority of honest parties. Asharov and Lindell [3] explained that in all the existing protocols, the designer needs to know the actual utility values of the players. They then showed that it is possible to achieve utility independence through the relaxation of assumptions. Gradwohl et al. [11] provided the definitions of computational solution concepts that guarantee sequential rationality. Finally, Asharov et al. [2] showed how game theoretic concepts can be used to capture cryptographic notions of security.

\section{Socio-Rational Secret Sharing}

We first provide formal definitions of a social game, a social Nash equilibrium, and socio-rational secret sharing. In our model, each $P_{i}$ has a public reputation value $\mathcal{T}_{i}$, where $\mathcal{T}_{i}(0)=0$ and $-1 \leq \mathcal{T}_{i}(p) \leq+1 ; p=0,1,2, \ldots$ denote the time periods of the games. The construction of this function is independent of our protocol, therefore, we use the existing function presented in Section 2.3. Since the importance of each game might be different, it would be possible to consider the transaction cost parameter (as stated in Section 2.3) during trust adjustment, but we do not use this in this paper. We assume each player's action $a_{i} \in\{\mathcal{C}, \mathcal{D}, \perp\}$, where $\mathcal{C}$ and $\mathcal{D}$ denote "cooperation" and "defection" respectively, and $\perp$ denotes $P_{i}$ has not been chosen by the dealer to participate in the current game.

Definition 6. In a society of size $N$, a social game $\Gamma=\left(\mathcal{A}_{i}, \mathcal{T}_{i}, u_{i}, u_{i}^{\prime}\right)$, where $1 \leq i \leq N$, is repeatedly played an unbounded number of times among different subsets of players. Each $P_{i}$ has a set of actions $\mathcal{A}_{i}$, a reputation value $\mathcal{T}_{i}$, a longterm utility function $u_{i}$, and an actual utility function $u_{i}^{\prime}$. Let $\mathcal{A} \stackrel{\text { def }}{=} \mathcal{A}_{1} \times \cdots \times \mathcal{A}_{N}$ be the action profile. In each game:

- A subset of $n \leq N$ players is chosen by the dealer for each new secret sharing game based on their reputation values $\mathcal{T}_{i}$, where more reputable players have a greater chance to be selected.

- Each $P_{i}$ estimates his long-term utility by $u_{i}: \mathcal{A} \times \mathcal{T}_{i} \mapsto \mathbb{R}$ based on his gain in the current game and future games. Player $P_{i}$ then selects his action $a_{i}$ according to $u_{i}$.

- Let $\boldsymbol{a}=\left(a_{1}, \ldots, a_{N}\right) \in \mathcal{A}$ be the current game's outcome. The actual utility of each $P_{i}$ is computed based on a function $u_{i}^{\prime}: \mathcal{A} \mapsto \mathbb{R}$ at the end of the current game.

- Each player's reputation value $\mathcal{T}_{i}$ is publicly updated by a trust function based on each player's action in the current game, as shown in Section 2.3, except that $\mathcal{T}_{i}(p)=\mathcal{T}_{i}(p-1)$ if $a_{i}=\perp$. 
Note that the long-term utility function $u_{i}$ is used for "action selection" and the actual utility function $u_{i}^{\prime}$ is used to compute the "real gain" at the end of the current game.

Definition 7. A vector of strategies $\boldsymbol{\sigma}$ is said to be a social Nash equilibrium in each game of a social game $\Gamma$ if for all $i$ and any $\sigma_{i}^{\prime} \neq \sigma_{i}$ it holds that $u_{i}\left(\sigma_{i}^{\prime}, \boldsymbol{\sigma}_{-i}\right) \leq u_{i}(\boldsymbol{\sigma})$. Accordingly, if $u_{i}\left(\sigma_{i}^{\prime}, \boldsymbol{\sigma}_{-i}\right)<u_{i}(\boldsymbol{\sigma})$, it is said to be a strict social Nash equilibrium. That is, considering future games, a player cannot gain any benefit by deviating from the protocol in the current game.

In the next sections, we discuss the utility function which is a central component in every game. This is due to the fact that players make decisions based on this function. Note that the utility assumption refers to the players' preferences over the game's outcome whereas the utility computation shows the method of computing the utility of each player.

\subsection{Utility Assumption}

Let $u_{i}(\boldsymbol{a})$ denotes $P_{i}$ 's utility resulting from a list of players' actions $\boldsymbol{a}$ by considering future games, let $u_{i}^{\prime}(\boldsymbol{a})$ denotes $P_{i}$ 's utility resulting from the current game, let $l_{i}(\boldsymbol{a}) \in\{0,1\}$ denote if $P_{i}$ has learned the secret during a given time period, and define $\delta(\boldsymbol{a})=\sum_{i} l_{i}(\boldsymbol{a})$. Also, let $\mathcal{T}_{i}^{a}(p)$ denote the reputation of $P_{i}$ after outcome $\boldsymbol{a}$ in period $p$; each game of a social game is played in a single period. The generalized assumptions of socio-rational secret sharing are as follows:

A. $l_{i}(\boldsymbol{a})=l_{i}\left(\boldsymbol{a}^{\prime}\right)$ and $\mathcal{T}_{i}^{\boldsymbol{a}}(p)>\mathcal{T}_{i}^{\boldsymbol{a}^{\prime}}(p) \Rightarrow u_{i}(\boldsymbol{a})>u_{i}\left(\boldsymbol{a}^{\prime}\right)$.

B. $l_{i}(\boldsymbol{a})>l_{i}\left(\boldsymbol{a}^{\prime}\right) \Rightarrow u_{i}^{\prime}(\boldsymbol{a})>u_{i}^{\prime}\left(\boldsymbol{a}^{\prime}\right)$.

C. $l_{i}(\boldsymbol{a})=l_{i}\left(\boldsymbol{a}^{\prime}\right)$ and $\delta(\boldsymbol{a})<\delta\left(\boldsymbol{a}^{\prime}\right) \Rightarrow u_{i}^{\prime}(\boldsymbol{a})>u_{i}^{\prime}\left(\boldsymbol{a}^{\prime}\right)$.

The preference " $A$ " illustrates that, whether player $P_{i}$ learns the secret or not, $P_{i}$ prefers to maintain a high reputation. The preferences " $B$ " and " $C$ " are the standard assumptions of rational secret sharing.

Definition 8. In a social game, a rational foresighted player has prioritized assumptions: "A" (greediness) is strictly preferred to " $B$ " and has an impact factor $\rho_{1}$, "B" (selfishness) is at least as good as " $C$ " and has an impact factor $\rho_{2}$, and " $C$ " (selfishness) has an impact factor $\rho_{3}$. We denote this using the notation $A^{\rho_{1}} \succ B^{\rho_{2}} \succeq C^{\rho_{3}}$, where $\rho_{1} \gg \rho_{2} \geq \rho_{3} \geq 1$.

The above definition reflects the fact that a rational foresighted player has a "long-term" vision and firstly prefers to achieve the highest level of trustworthiness. Only in this case, he will be involved in the future games and consequently gain more profits (interpreted as greediness). He secondly prefers an outcome in which he learns the secret. Finally, he desires the fewest number of other players learn the secret. We next propose a long-term utility function that satisfies all three preferences. 


\subsection{Utility Computation}

Our long-term utility function $u_{i}: \mathcal{A} \times \mathcal{T}_{i} \mapsto \mathbb{R}$ computes the utility that each player $P_{i}$ potentially gains or loses by considering future games, based on assumptions " $A$ ", " $B$ ", " $C$ ", whereas the actual utility function $u_{i}^{\prime}: \mathcal{A} \mapsto \mathbb{R}$ only computes the current gain or loss in a given time period, based on assumptions " $B$ " and " $C$ ".

Sample Function. We define two functions $\omega_{i}(\boldsymbol{a})$ and $\tau_{i}(\boldsymbol{a})$ for the $n$ participating players of the current game:

$$
\begin{aligned}
\omega_{i}(\boldsymbol{a}) & =\frac{3}{2-\mathcal{T}_{i}{ }^{a}(p)} \\
\tau_{i}(\boldsymbol{a}) & =\mathcal{T}_{i}{ }^{a}(p)-\mathcal{T}_{i}{ }^{a}(p-1) .
\end{aligned}
$$

Since $-1 \leq \mathcal{T}_{i}^{a}(p) \leq+1$, then $+1 \leq \omega_{i}(\boldsymbol{a}) \leq+3$. Let $\Omega>0$ be a "unit of utility", for instance, $\$ 100$. To satisfy our assumptions in Section 4.1, we define:

$$
\begin{aligned}
& A: \frac{\left|\tau_{i}(\boldsymbol{a})\right|}{\tau_{i}(\boldsymbol{a})} \times \omega_{i}(\boldsymbol{a}) \times \Omega \quad \text { where } \quad \frac{\left|\tau_{i}(\boldsymbol{a})\right|}{\tau_{i}(\boldsymbol{a})}=\left\{\begin{array}{l}
+1 \text { if } a_{i}=\mathcal{C} \\
-1 \text { if } a_{i}=\mathcal{D}
\end{array}\right. \\
& B: l_{i}(\boldsymbol{a}) \times \Omega \quad \text { where } \quad l_{i}(\boldsymbol{a}) \in\{0,1\} \\
& C: \frac{l_{i}(\boldsymbol{a})}{\delta(\boldsymbol{a})+1} \times \Omega \quad \text { where } \quad \delta(\boldsymbol{a})=\sum_{i=1}^{N} l_{i}(\boldsymbol{a}) .
\end{aligned}
$$

- (4) will evaluate to $+\omega_{i}(\boldsymbol{a}) \Omega$ if $P_{i}$ cooperates and it will evaluate to $-\omega_{i}(\boldsymbol{a}) \Omega$, otherwise. This means that $P_{i}$ gains or loses at least $1 \Omega$ and at most $3 \Omega$ (depending on his reputation value, as reflected in $\omega_{i}$ ) units of utility in the future games due to his current behavior.

- (5) illustrates that a player gains one unit of utility if he learns the secret in the current game and he loses this opportunity, otherwise.

- (6) results in "almost" one unit of utility being divided among all the players $P_{i}$ who have learned the secret in the current game; to avoid a division by 0 when $\delta(\boldsymbol{a})=0$, we use $\delta(\boldsymbol{a})+1$ in the denominator.

We combine these three terms, weighted with their corresponding impact factors:

$$
\begin{gathered}
u_{i}^{\prime}(\boldsymbol{a})=\rho_{2}\left(l_{i}(\boldsymbol{a}) \times \Omega\right)+\rho_{3}\left(\frac{l_{i}(\boldsymbol{a})}{\delta(\boldsymbol{a})+1} \times \Omega\right), \text { and } \\
u_{i}(\boldsymbol{a})=\rho_{1}\left(\frac{\left|\tau_{i}(\boldsymbol{a})\right|}{\tau_{i}(\boldsymbol{a})} \times \omega_{i}(\boldsymbol{a}) \times \Omega\right)+u_{i}^{\prime}(\boldsymbol{a}) \\
=\Omega \times\left(\rho_{1}\left(\frac{\left|\tau_{i}(\boldsymbol{a})\right|}{\tau_{i}(\boldsymbol{a})} \times \omega_{i}(\boldsymbol{a})\right)+\rho_{2}\left(l_{i}(\boldsymbol{a})\right)+\rho_{3}\left(\frac{l_{i}(\boldsymbol{a})}{\delta(\boldsymbol{a})+1}\right)\right) .
\end{gathered}
$$


The function $u_{i}(\boldsymbol{a})$ shows that if player $P_{i}$, with preference factors $\rho_{1} \gg \rho_{2} \geq$ $\rho_{3} \geq 1$, defects (or cooperates), he may gain (or lose) $\rho_{2} \Omega+\left(\rho_{3} \Omega\right) /(\delta(\boldsymbol{a})+1)$ utility in the current game, but he will lose (or gain) " $x$ " units of utility in the future games, where $\rho_{1} \Omega \leq x \leq 3 \rho_{1} \Omega$. That is, future loss or gain is more important than the current loss or gain. We later show that the dealer gives a lesser (or a greater) chance of contribution to non-reputable (or reputable) players in the future games, that is, reputation remains with a player as a characteristic which continuously affects his utility.

\subsection{Proposed Protocol}

We now discuss our socio-rational secret sharing scheme, the details are presented in Figure 4. Suppose the public trust network has already been created. Assume we have a dealer who initiates a $(t, n)$-threshold secret sharing scheme. Also, assume all the players use a "pure-strategy". A socio-rational secret sharing game $\Gamma=\left(\mathcal{A}_{i}, \mathcal{T}_{i}, u_{i}, u_{i}^{\prime}\right)$ is a social game that is played among rational foresighted players and it is based on the following elements:

1. Set of possible actions $\mathcal{A}_{i}=\{\mathcal{C}, \mathcal{D}, \perp\}$, defined in Section 4 .

2. Function $\mathcal{T}_{i}$, except that $\mathcal{T}_{i}(p)=\mathcal{T}_{i}(p-1)$ if $a_{i}=\perp$, defined in Section 2.3.

3. Long-term utility function $u_{i}: \mathcal{A} \times \mathcal{T}_{i} \mapsto \mathbb{R}$, defined in Section 4.2.

4. Actual utility function $u_{i}^{\prime}: \mathcal{A} \mapsto \mathbb{R}$, defined in Section 4.2.

Secret Sharing

1. Let $\phi$ be the current probability distribution over players' types $\mathcal{B}, \mathcal{N}, \mathcal{G}$, as defined in Section 2.3. The dealer $D$ selects $n$ out of $N$ players, where $n \leq N$, based on this non-uniform probability distribution.

2. $D$ then initiates a $(t, n)$-secret sharing scheme by selecting $f(x) \in \mathbb{Z}_{q}[x]$ of degree $t-1$, where $f(0)=\alpha$ is the secret. Subsequently, he sends shares $f(i)$ to $P_{i}$ for the $n$ chosen players, and leaves the scheme.

$\underline{\text { Secret Recovery }}$

1. Each chosen player $P_{i}$ computes his long-term utility function $u_{i}: \mathcal{A} \times \mathcal{T}_{i} \mapsto \mathbb{R}$, and then selects an action, i.e., revealing or not revealing his share $f(i)$.

2. If enough shares are revealed, the polynomial $f(x)$ is reconstructed through Lagrange interpolation and the secret $f(0)=\alpha$ is recovered.

3. Each chosen player $P_{i}$ receives his utility $u_{i}^{\prime}: \mathcal{A} \mapsto \mathbb{R}$ (i.e., the real payment) at the end of the reconstruction phase according to the outcome.

4. Finally, the reputation values $\mathcal{T}_{i}$ of all the chosen players are publicly updated according to each player's behavior and the trust function.

Fig. 4. Socio-Rational Secret Sharing Protocol 
The sharing phase is similar to that of standard secret sharing. The only difference is the way that the dealer selects $n$ out of $N$ players for secret sharing. In other words, the dealer gives more chance to reputable players compared to unreliable parties. Although a natural approach is to invite only the reputable players, it is not fair if the dealer does not provide any opportunity for newcomers, or if he completely ignores the bad players. Once in a while, he should give a chance to the bad players so they can compensate for their past behavior. This is a realistic approach even in human society; it can be interpreted as a "forgiveness factor". The secret recovery phase is also similar to that of the standard secret sharing but with some extra components.

We should mention that since the players' reputations and the trust function are public information. Therefore, all computations associated with the reputation system can be done by any authority or a committee of the players. It is also worth mentioning that it is not required to consider unknown number of iterations for secret recovery, which is the case in all the existing rational secret sharing schemes. In fact, in a "socio-rational secret sharing" game, we have an unknown number of independent secret sharing games, whereas in "rational secret sharing", we only have one secret with an unknown number iterations for secret recovery.

Theorem 1. In a $(2,2)$-socio-rational secret sharing, $\mathcal{C}$ strictly dominates $\mathcal{D}$, considering a long-term utility function, shown in Equation (8), which satisfies the preferences of rational foresighted players, shown in Definition 8. In other words, $\mathcal{D}$ is strictly dominated by $\mathcal{C}$. As a result, $(\mathcal{C}, \mathcal{C})$ is a strict social Nash equilibrium that is a unique solution.

Proof. We compute the utility of each outcome for $P_{i}$. Let $P_{j}$ be the other player.

1. If both players cooperate, denoted by $(\mathcal{C}, \mathcal{C})$, then $\tau_{i}$ is positive, $l_{i}=1$ since $P_{i}$ has learned the secret, and $\delta=2$ because both players have learned the secret. We have:

$$
\left(\tau_{i}>0, l_{i}=1, \delta=2\right) \Rightarrow u_{i}^{(\mathcal{C}, \mathcal{C})}(\boldsymbol{a})=\Omega\left(\rho_{1} \omega_{i}+\rho_{2}+\frac{\rho_{3}}{3}\right)
$$

2. If only $P_{i}$ cooperates, denoted by $(\mathcal{C}, \mathcal{D})$, then $\tau_{i}$ is positive, $l_{i}=0$ since $P_{i}$ has not learned the secret, and $\delta=1$ because only player $P_{j}$ has learned the secret. We have:

$$
\left(\tau_{i}>0, l_{i}=0, \delta=1\right) \Rightarrow u_{i}^{(\mathcal{C}, \mathcal{D})}(\boldsymbol{a})=\Omega\left(\rho_{1} \omega_{i}\right)
$$

3. If only $P_{j}$ cooperates, denoted by $(\mathcal{D}, \mathcal{C})$, then $\tau_{i}$ is negative, $l_{i}=1$ since $P_{i}$ has learned the secret, and $\delta=1$ because only player $P_{i}$ has learned the secret. We have:

$$
\left(\tau_{i}<0, l_{i}=1, \delta=1\right) \Rightarrow u_{i}^{(\mathcal{D}, \mathcal{C})}(\boldsymbol{a})=\Omega\left(-\rho_{1} \omega_{i}+\rho_{2}+\frac{\rho_{3}}{2}\right) .
$$


4. If both players defect, denoted by $(\mathcal{D}, \mathcal{D})$, then $\tau_{i}$ is negative, $l_{i}=0$ since $P_{i}$ has not learned the secret, and $\delta=0$ because no one has learned the secret. We have:

$$
\left(\tau_{i}<0, l_{i}=0, \delta=0\right) \Rightarrow u_{i}^{(\mathcal{D}, \mathcal{D})}(\boldsymbol{a})=\Omega\left(-\rho_{1} \omega_{i}\right)
$$

We ignore the common factor $\Omega$. We know $1 \leq \omega_{i}(\boldsymbol{a}) \leq 3$ and $\rho_{1} \gg \rho_{2} \geq \rho_{3} \geq 1$.

- First, we have:

$$
u_{i}^{(\mathcal{C}, \mathcal{C})}(\boldsymbol{a})=\rho_{1} \omega_{i}+\rho_{2}+\frac{\rho_{3}}{3}>\rho_{1} \omega_{i}=u_{i}^{(\mathcal{C}, \mathcal{D})}(\boldsymbol{a})
$$

- Next, it is easy to see that

$$
u_{i}^{(\mathcal{C}, \mathcal{D})}(\boldsymbol{a})=\rho_{1} \omega_{i}>-\rho_{1} \omega_{i}+\rho_{2}+\frac{\rho_{3}}{2}=u_{i}^{(\mathcal{D}, \mathcal{C})}(\boldsymbol{a})
$$

if and only if $2 \rho_{1} \omega_{i}>\rho_{2}+\frac{\rho_{3}}{2}$. We have:

$$
\begin{aligned}
2 \rho_{1} \omega_{i} & \geq 2 \rho_{1} \\
& >\rho_{2}+\rho_{3} \\
& >\rho_{2}+\frac{\rho_{3}}{2},
\end{aligned}
$$

so the desired conclusion follows.

- Finally,

$$
u_{i}^{(\mathcal{D}, \mathcal{C})}(\boldsymbol{a})=-\rho_{1} \omega_{i}+\rho_{2}+\frac{\rho_{3}}{2}>-\rho_{1} \omega_{i}=u_{i}^{(\mathcal{D}, \mathcal{D})}(\boldsymbol{a}) .
$$

Therefore, we have the following payoff inequalities which proves the theorem:

$$
\overbrace{u_{i}^{(\mathcal{C}, \mathcal{C})}(\boldsymbol{a})>u_{i}^{(\mathcal{C}, \mathcal{D})}(\boldsymbol{a})}^{P_{i} \text { cooperates }}>\overbrace{u_{i}^{(\mathcal{D}, \mathcal{C})}(\boldsymbol{a})>u_{i}^{(\mathcal{D}, \mathcal{D})}(\boldsymbol{a})}^{P_{i} \text { defects }} .
$$

The interesting observation is the difference between the utilities $u_{i}^{(\mathcal{C}, \mathcal{D})}(\boldsymbol{a})$ and $u_{i}^{(\mathcal{D}, \mathcal{C})}(\boldsymbol{a})$. This means that it is better for player $P_{i}$ to cooperate, even though he might not learn the secret and the other party might learn it. On the other hand, even if $P_{i}$ learns the secret by deviating at a given period (using the share of the other party), he will gain less utility in the long-term. This is due to future gain or loss and the significance of being reputable, which is incorporated in our long-term utility function by considering an impact factor $\rho_{1}$. We should also note that, as $\rho_{1}$ is increased, the difference between $u_{i}^{(\mathcal{C}, \mathcal{D})}(\boldsymbol{a})$ and $u_{i}^{(\mathcal{D}, \mathcal{C})}(\boldsymbol{a})$ also increases, i.e., the enforcement for cooperation would be greater.

In a secret sharing scheme with selfish players, the outcome $\left(\mathcal{U}^{-}, \mathcal{U}^{-}\right)$is a Nash equilibrium, as shown in Table 3 , where $\mathcal{U}^{+}>\mathcal{U}>\mathcal{U}^{-}>\mathcal{U}^{--}$. Rational secret sharing solves this problem by using a randomized mechanism, as presented in Section 2.2. The payoff matrix associated with socio-rational secret 


\begin{tabular}{|c|c|c|}
\hline$P_{1} P_{2}$ & Coope & Defection \\
\hline Cooperation & $\mathcal{U}, \mathcal{U}$ & $\mathcal{U}^{--}, \mathcal{U}^{+}$ \\
\hline Defection & $\mathcal{U}^{+}, \mathcal{U}^{--}$ & $\mathcal{U}^{-}, \mathcal{U}^{-}$ \\
\hline
\end{tabular}

Table 3. (2,2)-SS with Selfish Players

\begin{tabular}{|c|c|c|}
\hline$P_{1} P_{2}$ & Cooperation & Defection \\
\hline Cooperation & $\overline{\mathcal{U}^{+}, \mathcal{U}^{+}}$ & $\mathcal{U}, \mathcal{U}^{-}$ \\
\hline Defection & $\mathcal{U}^{-}, \mathcal{U}$ & $\mathcal{U}^{--}, \mathcal{U}^{--}$ \\
\hline
\end{tabular}

Table 4. (2, 2)-Socio-Rational SS

sharing is illustrated in Table 4 . In this payoff matrix, the outcome $\left(\mathcal{U}^{+}, \mathcal{U}^{+}\right)$is a strict social Nash equilibrium.

We should note that our socio-rational game is a non-cooperative game. In fact, cooperation is self-enforcing due to the importance of reputation as well as future concerns of a rational foresighted player. In a cooperative game, this enforcement is provided by a third party and players do not really compete. Moreover, this payoff matrix does not mean that the players never deviate. As an example, consider a scenario in which a player is involved in many independent social games. If he simultaneously receives many requests for secret recovery of various schemes, he will select the one in which he can gain more utility. This is discussed later, in Section 4.4.

We now analyze our socio-rational secret sharing in a setting where $n>2$ players take part in each secret sharing game.

Theorem 2. In a socio-rational secret sharing scheme with $n$ participants and $t=2, \mathcal{C}$ strictly dominates $\mathcal{D}$ for all $P_{i}$, assuming the preferences of rational foresighted parties. Consequently, the vector $\boldsymbol{a}^{\mathcal{C}}=\left(a_{1}^{\mathcal{C}}, \ldots, a_{n}^{\mathcal{C}}\right)$ is a strict social Nash equilibrium that is a unique solution.

Proof. Let $\mathcal{C}_{i}$ (or $\mathcal{D}_{i}$ ) denote that player $P_{i}$ cooperates (or defects), and let $\mathcal{C}_{-i}$ (or $\mathcal{D}_{-i}$ ) denote that, excluding $P_{i}$, all the other players cooperate (or defect), and finally let $\mathcal{M}_{-i}$ denotes that, excluding $P_{i}$, some players cooperate and some of them defect, that is, we have both $\mathcal{C}$ ooperation and $\mathcal{D}$ efection. We compute the utility of each outcome based on Equation (8) for the least possible threshold $t=2$ when $n>2$, i.e., two shares are enough to learn any secret.

1. If all the players cooperate, denoted by $\left(\mathcal{C}_{i}, \mathcal{C}_{-i}\right)$, then $\tau_{i}$ is positive, $l_{i}=1$ since player $P_{i}$ has learned the secret, and $\delta=n$ because all the players have learned the secret. We have:

$$
\left(\tau_{i}>0, l_{i}=1, \delta=n\right) \Rightarrow u_{i}^{\left(\mathcal{C}_{i}, \mathcal{C}_{-i}\right)}(\boldsymbol{a})=\Omega\left(\rho_{1} \omega_{i}+\rho_{2}+\frac{\rho_{3}}{n+1}\right) .
$$

2. If player $P_{i}$ cooperates but some of the other parties cooperate and some defect, denoted by $\left(\mathcal{C}_{i}, \mathcal{M}_{-i}\right)$, then $\tau_{i}$ is positive, $l_{i}=1$, and $\delta=n$ because all the players have learned the secret. We have:

$$
\left(\tau_{i}>0, l_{i}=1, \delta=n\right) \Rightarrow u_{i}^{\left(\mathcal{C}_{i}, \mathcal{M}_{-i}\right)}(\boldsymbol{a})=\Omega\left(\rho_{1} \omega_{i}+\rho_{2}+\frac{\rho_{3}}{n+1}\right) .
$$


3. If only $P_{i}$ cooperates, denoted by $\left(\mathcal{C}_{i}, \mathcal{D}_{-i}\right)$, then $\tau_{i}$ is positive, $l_{i}=0$, and $\delta=n-1$ because all the players, except $P_{i}$, have learned the secret. We have:

$$
\left(\tau_{i}>0, l_{i}=0, \delta=n-1\right) \Rightarrow u_{i}^{\left(\mathcal{C}_{i}, \mathcal{D}_{-i}\right)}(\boldsymbol{a})=\Omega\left(\rho_{1} \omega_{i}\right)
$$

4. If only $P_{i}$ defects, denoted by $\left(\mathcal{D}_{i}, \mathcal{C}_{-i}\right)$, then $\tau_{i}$ is negative, $l_{i}=1$, and $\delta=n$ because all the players have learned the secret. We have:

$$
\left(\tau_{i}<0, l_{i}=1, \delta=n\right) \Rightarrow u_{i}^{\left(\mathcal{D}_{i}, \mathcal{C}_{-i}\right)}(\boldsymbol{a})=\Omega\left(-\rho_{1} \omega_{i}+\rho_{2}+\frac{\rho_{3}}{n+1}\right) .
$$

5. If player $P_{i}$ defects but some of the other parties cooperate and some defect, denoted by $\left(\mathcal{D}_{i}, \mathcal{M}_{-i}\right)$, then $\tau_{i}$ is negative, $l_{i}=1$, and $\delta=n-1$ if only one player reveals his share, or $\delta=n$ if at least two players reveal their shares. We have:

$$
\left(\tau_{i}<0, l_{i}=1, \delta\right) \Rightarrow u_{i}^{\left(\mathcal{D}_{i}, \mathcal{M}_{-i}\right)}(\boldsymbol{a})=\Omega\left(-\rho_{1} \omega_{i}+\rho_{2}+\frac{\rho_{3}}{\delta+1}\right)
$$

where $\delta \in\{n-1, n\}$.

6. If all the players defect, denoted by $\left(\mathcal{D}_{i}, \mathcal{D}_{-i}\right)$, then $\tau_{i}$ is negative, $l_{i}=0$, and $\delta=0$ because no one has learned the secret. We have:

$$
\left(\tau_{i}<0, l_{i}=0, \delta=0\right) \Rightarrow u_{i}^{\left(\mathcal{D}_{i}, \mathcal{D}_{-i}\right)}(\boldsymbol{a})=\Omega\left(-\rho_{1} \omega_{i}\right) .
$$

We now analyze these six scenarios:

- If player $P_{i}$ cooperates (cases $1-3$ ), regardless of whether the other players cooperate or defect, then

$$
u_{i}^{\mathcal{C}}(\boldsymbol{a}) \geq \rho_{1} \omega_{i}
$$

- If $P_{i}$ defects (cases $4-6$ ), regardless of whether the other players cooperate or defect, then

$$
u_{i}^{\mathcal{D}}(\boldsymbol{a}) \leq-\rho_{1} \omega_{i}+\rho_{2}+\frac{\rho_{3}}{n} .
$$

It is easy to prove that $\rho_{1} \omega_{i}>-\rho_{1} \omega_{i}+\rho_{2}+\frac{\rho_{3}}{n}$. In fact, the proof is essentially the same as the proof of (10) in Theorem 1 . As a result, it is always in $P_{i}$ 's best interest to cooperate:

$$
u_{i}^{\mathcal{C}}(\boldsymbol{a})>u_{i}^{\mathcal{D}}(\boldsymbol{a})
$$

Remark 1. A similar analysis can be given for any threshold $t>2$ with any number of players. 


\subsection{Expected Utility}

In this section, we illustrate how each $P_{i}$ can compute his expected utility when he participates in different independent social games. Note that the utility value shows the connection between actions and their corresponding consequences for a player, whereas the expected utility of $P_{i}$ is an estimation of gain or loss when he plays with another player $P_{j}$.

We initially show how to compute the expected utilities in a $(2,2)$-game for "cooperation" and "defection". An expected utility is computed as a linear combination of utility values and the probability of $P_{j}$ 's cooperation, where $\epsilon_{j} \in[0,1]$ denotes the probability that the opponent $P_{j}$ may cooperate and $\mathcal{U}^{+}>\mathcal{U}>\mathcal{U}^{-}>\mathcal{U}^{--}$are the utility values from Table 4 . We have:

$$
\begin{aligned}
& \mathcal{E} \mathcal{U}_{i}^{\mathcal{C}}(\boldsymbol{a})=\epsilon_{j} \mathcal{U}^{+}+\left(1-\epsilon_{j}\right) \mathcal{U} \\
& \mathcal{E U}_{i}^{\mathcal{D}}(\boldsymbol{a})=\epsilon_{j} \mathcal{U}^{-}+\left(1-\epsilon_{j}\right) \mathcal{U}^{--}
\end{aligned}
$$

Theorem 3. In a socio-rational secret sharing game with two players $P_{i}$ and $P_{j}$, the expected utility of cooperation is greater than the expected utility of defection, i.e., $\mathcal{E U}_{i}^{\mathcal{C}}(\boldsymbol{a})-\mathcal{E U}_{i}^{\mathcal{D}}(\boldsymbol{a})>0$, where $\epsilon_{j}$ is the probability of opponent's cooperation.

Proof.

$$
\begin{aligned}
\mathcal{E U}_{i}^{\mathcal{C}}(\boldsymbol{a})-\mathcal{E U}_{i}^{\mathcal{D}}(\boldsymbol{a}) & =\left(\epsilon_{j} \mathcal{U}^{+}+\left(1-\epsilon_{j}\right) \mathcal{U}\right)-\left(\epsilon_{j} \mathcal{U}^{-}+\left(1-\epsilon_{j}\right) \mathcal{U}^{--}\right) \text {by }(14,15) \\
& =\epsilon_{j}\left(\mathcal{U}^{+}-\mathcal{U}^{-}\right)+\left(1-\epsilon_{j}\right)\left(\mathcal{U}-\mathcal{U}^{--}\right) \\
& >0
\end{aligned}
$$

We now consider the expected utilities in two independent $(2,2)$-games. Let us define $\mathcal{E U}_{i}^{\mathcal{C}}\left(\boldsymbol{a}_{i j}\right)$ and $\mathcal{E U}_{i}^{\mathcal{C}}\left(\boldsymbol{a}_{i k}\right)$ as the expected utilities of the two games, when player $P_{i}$ cooperates with players $P_{j}$ and $P_{k}$ respectively.

Theorem 4. Suppose $P_{i}$ plays with $P_{j}$ and $P_{k}$ in two independent $(2,2)$-games. Player $P_{i}$ then gains more utility if he collaborates with the most reputable player.

Proof. Let $P_{j}$ and $P_{k}$ have different reputation values computed with the same trust function. For instance, $\epsilon_{j}>\epsilon_{k}$, which means $P_{j}$ is more reputable than $P_{k}$. Suppose $P_{i}$ receives the same unit of utility $\Omega$ in both games, and let $\boldsymbol{a}_{i j}, \boldsymbol{a}_{i k}$ be the outcomes of the two games. We have:

$$
\begin{aligned}
\mathcal{E U}_{i}^{\mathcal{C}}\left(\boldsymbol{a}_{i j}\right)-\mathcal{E U}_{i}^{\mathcal{C}}\left(\boldsymbol{a}_{i k}\right) & =\left(\epsilon_{j} \mathcal{U}^{+}+\left(1-\epsilon_{j}\right) \mathcal{U}\right)-\left(\epsilon_{k} \mathcal{U}^{+}+\left(1-\epsilon_{k}\right) \mathcal{U}\right) \quad \text { by } \\
& =\epsilon_{j} \mathcal{U}^{+}-\epsilon_{k} \mathcal{U}^{+}+\left(1-\epsilon_{j}\right) \mathcal{U}-\left(1-\epsilon_{k}\right) \mathcal{U} \\
& =\left(\epsilon_{j}-\epsilon_{k}\right) \mathcal{U}^{+}+\left(\epsilon_{j}-\epsilon_{k}\right) \mathcal{U} \\
& >0
\end{aligned}
$$

since $\epsilon_{j}>\epsilon_{k}$. As a result, $\mathcal{E U}_{i}^{\mathcal{C}}\left(\boldsymbol{a}_{i j}\right)>\mathcal{E} \mathcal{U}_{i}^{\mathcal{C}}\left(\boldsymbol{a}_{i k}\right)$. This means that player $P_{i}$ gains more utility if he collaborates with $P_{j}$ rather than $P_{k}$. 


\section{Comparison with Existing Techniques}

Our contribution differs from rational secret sharing and social secret sharing, as shown in Figure 5. Our scheme is a repeated game that addresses the problem of secret recovery in the presence of rational foresighted parties, whereas:

- "rational secret sharing" is a one-time game with repeated rounds, and it deals with the problem of secret recovery of a secret in the presence of rational players, and

- "social secret sharing" defines how many shares each player can hold in a weighted secret sharing scheme with honest and malicious parties.

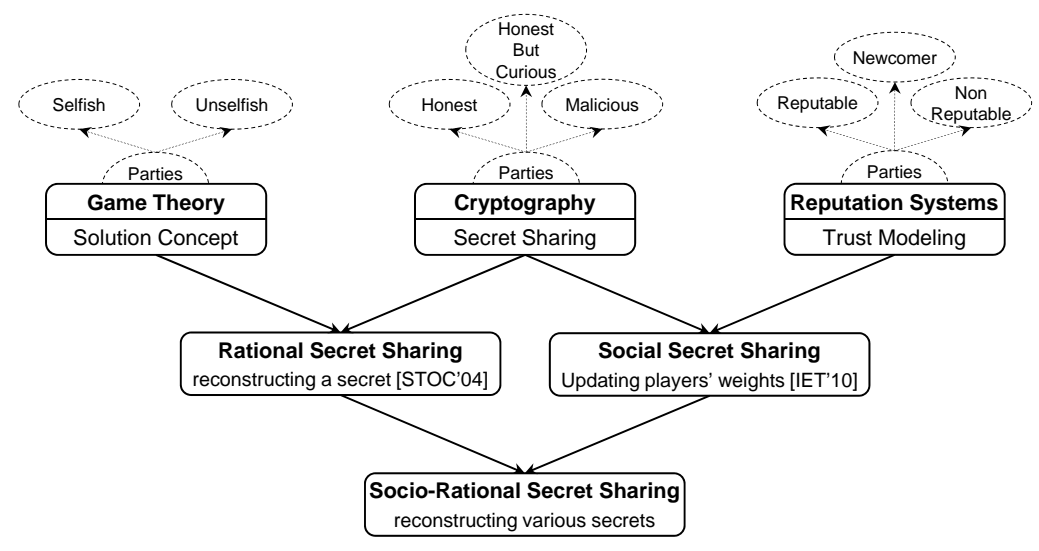

Fig. 5. Pedigree of the Socio-Rational Secret Sharing

Our contribution is also different from the punishment strategy used in the repeated prisoners' dilemma [28] where the players penalize potential deviants. As the authors have mentioned, the major point behind the repeated games is the fact that if each participant believes any deviation terminates the mutual cooperation (resulting in a subsequent loss that outweighs the short-term gain), he then prefers to cooperate. For instance, consider the prisoners' dilemma with $\mathcal{C}$ ooperation and Defection actions. Both players cooperate until one of them deviates. Then, the other player chooses $\mathcal{D}$ for a specific number of times as a punishment. Meanwhile, the deviant rewards the punisher by selecting $\mathcal{C}$ as a compensation. Finally, the game returns to a mutual cooperation. Our approach has the following advantages over the punishment strategy:

- In our model, a player is not just an abstract entity who selects actions. He also has a social characteristic reflected in his reputation that shows his trustworthiness. This attribute is solely determined by the player's actions. 
- The punishment strategy is performed by selecting actions that are harmful for deviants whereas, in our model, punishment or reward (losing or gaining reputation and utility) is independent of action selection.

- Our approach avoids penalizing innocent players or the punisher himself. It also avoids being involved, to some extent, in a game with seriously selfish players who are not reputable (due to our "invitation approach").

- The punishment strategy does not consider that a game may have various levels of importance and utility weights when it is repeatedly played. For instance, whether it is a secret sharing scheme to launch a "missile" or to open a "safety box".

- The punishment strategy has a discrete penalizing approach whereas our construction has a continuous impact on the deviants. For example, it may take a long time for a player to regain lost reputation.

- Our proposed approach not only considers punishment and reward but also defines six different scenarios in order to fairly deal with various types of players, including good players, bad players, and newcomers.

Our contribution is also different from the constructions forming histories and beliefs such as subgame perfect equilibrium or Bayesian equilibrium [28]. In the former, players reassess their decisions based on the past history, i.e., a sequence of previous actions. In the latter, the game is designed to deal with the situations in which parties are not certain about the characteristics of each others. Therefore, they form beliefs, i.e., a probability distributions over actions, to anticipate any future behavior.

Let $P_{i}$ be a specific player, and let $P_{j}$ for $1 \leq j \neq i \leq n$ denote any other player except $P_{i}$. Our trust calculation method and social setting differs from these kinds of solution concepts in the following aspects:

- In forming a belief about $P_{i}$ 's intentions, both parties contribute. That is, $P_{i}$ is indirectly involved by his behavior, i.e., action selections, and the other players are directly involved by the methodology that they use in order to form the probability distribution over actions. A belief may or may not be common knowledge, meaning that various players may have different judgments and beliefs about $P_{i}$. On the other hand, the reputation of $P_{i}$ in a trust network is solely determined by his behavior through a trust function, which is a commonly known function for reputation measurement. That is, the reputation is a direct reflection of $P_{i}$ 's attitude (there is no misunderstanding), and he knows the impact of his decision on the other players (i.e., whether he is known as a good player, a bad player, or a newcomer). He can also estimate how much extra utility he may gain or lose after his reputation's adjustment, which is a strong enforcement.

- Histories and beliefs are more general compared to the reputation system in a trust network. This means a belief as a probability distribution can be defined over any set of actions for any types of players. On the other hand, reputation is built over a specific set of actions, such as Cooperation and $\mathcal{D}$ efection $(\mathcal{X}$ : corruption as a malicious behavior might be also considered 
in a mixed model), for specific types of players, such as good players, bad players, and newcomers. As a result, the reputation system is simpler and it is more suitable for cryptographic constructions.

- In the history and belief systems, all the measurements are "inside" the game-theoretic model whereas our reputation system isolates these computations from the game. For instance, two separate probability distributions can be defined over the players' types and actions by considering their past behavior ${ }^{3}$. But our publicly known trust function combines these two measurements in a single reputation value outside of the game-theoretic model (although these values might be interpreted similar to "types" and "beliefs"). In other words, the punishment or reward is embedded inside of our reputation system which continuously affects the players' utilities in the gametheoretic model, i.e., losing utility due to the reputation's decline or losing reputation and not being selected in the future secret sharing games.

\section{Conclusion and Future Direction}

This paper provides a multidisciplinary research connecting three major areas of computer science in order to propose a novel solution for one of the most fundamental cryptographic primitives.

As we illustrated, the social network with reputation consideration is a strong enforcement for the participants to cooperate, for instance, a player may change his non-cooperative approach after analyzing his reputation, or after estimating his future loss. In our social setting, bad players can compensate for their past behavior by continuous cooperation. On the other hand, reputable players can gain more profits as long as they act properly, and newcomers can fairly start their social interactions. Finally, we should stress that having a trust network by considering long-term interactions can be seen as a new direction in game theory itself, specifically, the theoretical models used in social sciences such as economics and political science because elements in those frameworks are more close to human social behavior.

As our future work, we are interested to consider other complicated models. For instance, using referral chain in which two players who are interacting for the first time, can gain some information with respect to each other's reputation through other parties or common friends. We also would like to scrutinize the impact of a situation in which a player is involved in various societies while he is holding different reputation values associated with each one. It would be also interesting to construct a hybrid model in which both "reputation" and "belief" are considered. In this case, reputation can be seen as an estimation of the past behavior whereas belief can be viewed as an anticipation of the future activities.

\footnotetext{
${ }^{3}$ For instance, suppose that $P_{i}$ is good or bad with probabilities 0.7 or 0.3 respectively. Based on these values, a $P_{j}$ may believe that $P_{i}$ reveals or denies to reveal his share with certain probabilities, e.g., 0.9 or 0.1 respectively.
} 


\section{References}

1. Abraham, I., Dolev, D., Gonen, R., and Halpern, J. Y. Distributed computing meets game theory: robust mechanisms for rational secret sharing and multiparty computation. In 25th Annual ACM Symposium on Principles of Distributed Computing PODC (2006), pp. 53-62.

2. Asharov, G., Canetti, R., and Hazay, C. Towards a game theoretic view of secure computation. In 30th Annual International Conference on the Theory and Applications of Cryptographic Techniques EUROCRYPT (2011), vol. 6632 of LNCS, Springer, pp. 426-445.

3. Asharov, G., and Lindell, Y. Utility dependence in correct and fair rational secret sharing. In 29th Annual International Cryptology Conference CRYPTO (2009), vol. 5677 of $L N C S$, Springer, pp. 559-576.

4. Ben-Or, M., Goldwasser, S., And Wigderson, A. Completeness theorems for non-cryptographic fault-tolerant distributed computation. In 20th Annual ACM Symposium on Theory of Computing STOC (1988), pp. 1-10.

5. Blakley, G. Safeguarding cryptographic keys. In Proc. NCC (1979), vol. 48, AFIPS Press, pp. 313-317.

6. Chaum, D., Crépeau, C., and Damgård, I. Multiparty unconditionally secure protocols. In 20th Annual ACM Symposium on Theory of Computing STOC (1988), pp. 11-19.

7. Dodis, Y., Halevi, S., And Rabin, T. A cryptographic solution to a game theoretic problem. In 20th Annual International Cryptology Conference CRYPTO (2000), vol. 1880 of $L N C S$, Springer, pp. 112-130.

8. Fuchsbauer, G., Katz, J., And NacCache, D. Efficient rational secret sharing in standard communication networks. In 7th Theory of Cryptography Conference TCC (2010), vol. 5978 of $L N C S$, Springer, pp. 419-436.

9. Goldreich, O., Micali, S., And Wigderson, A. How to play any mental game or a completeness theorem for protocols with honest majority. In 19th Annual ACM Symposium on Theory of Computing STOC (1987), pp. 218-229.

10. Gordon, S. D., And KATz, J. Rational secret sharing, revisited. In 5th International Conference on Security and Cryptography for Networks SCN (2006), vol. 4116 of $L N C S$, Springer, pp. 229-241.

11. Gradwohl, R., Livne, N., And Rosen, A. Sequential rationality in cryptographic protocols. In 51th Annual IEEE Symposium on Foundations of Computer Science FOCS (2010), pp. 623-632.

12. Halpern, J. Y., and Teague, V. Rational secret sharing and multiparty computation: extended abstract. In 36th Annual ACM Symposium on Theory of Computing STOC (2004), pp. 623-632.

13. Harkavy, M., Tygar, J. D., AND KIKUChi, H. Electronic auctions with private bids. In 3rd Conference on USENIX Workshop on Electronic Commerce WOEC (1998), USENIX Association, pp. 61-74.

14. Izmalkov, S., Micali, S., AND LePinski, M. Rational secure computation and ideal mechanism design. In 46th Annual IEEE Symposium on Foundations of Computer Science FOCS (2005), pp. 585-595.

15. KATZ, J. Bridging game theory and cryptography: Recent results and future directions. In 5th Theory of Cryptography Conference TCC (2008), vol. 4948 of LNCS, Springer, pp. 251-272.

16. KOL, G., AND NAOR, M. Cryptography and game theory: Designing protocols for exchanging information. In 5th Theory of Cryptography Conference TCC (2008), vol. 4948 of $L N C S$, Springer, pp. 320-339. 
17. Kol, G., AND NAOr, M. Games for exchanging information. In 40th Annual ACM Symposium on Theory of Computing STOC (2008), pp. 423-432.

18. Lepinski, M., Micali, S., Peikert, C., and Shelat, A. Completely fair SFE and coalition-safe cheap talk. In 23th Annual ACM Symposium on Principles of Distributed Computing PODC (2004), pp. 1-10.

19. Lepinski, M., Micali, S., And Shelat, A. Collusion-free protocols. In 37th Annual ACM Symposium on Theory of Computing STOC (2005), pp. 543-552.

20. Lysyanskaya, A., And Triandopoulos, N. Rationality and adversarial behavior in multi-party computation. In 26th International Cryptology Conference CRYPTO (2006), vol. 4117 of LNCS, Springer, pp. 180-197.

21. Mailath, G., AND Samuelson, L. Repeated games and reputations: long-run relationships. Oxford University Press, USA, 2006.

22. Maleka, S., Shareef, A., And Rangan, C. P. Rational secret sharing with repeated games. In 4 th Int. Conf. on Information Security Practice and Experience ISPEC (2008), vol. 4991 of LNCS, Springer, pp. 334-346.

23. Micali, S., And Shelat, A. Purely rational secret sharing. In 6th Theory of Cryptography Conference TCC (2009), vol. 5444 of LNCS, Springer, pp. 54-71.

24. Nojoumian, M., And Lethbridge, T. A New Approach for the Trust Calculation in Social Networks. In E-business and Telecommunication Networks: 3rd Int. Conf. on E-Business ICE-B'06, Best Papers (2008), vol. 9, Springer, pp. 64-77.

25. Nojoumian, M., Stinson, D., And Grainger, M. Unconditionally secure social secret sharing scheme. IET Information Security, Special Issue on Multi-Agent and Distributed Information Security 4, 4 (2010), 202-211.

26. Nojoumian, M., And Stinson, D. R. Brief announcement: secret sharing based on the social behaviors of players. In 29th ACM symposium on Principles of distributed computing PODC (2010), pp. 239-240.

27. Ong, S. J., Parkes, D. C., Rosen, A., And Vadhan, S. P. Fairness with an honest minority and a rational majority. In 6th Theory of Cryptography Conference TCC (2009), vol. 5444 of $L N C S$, Springer, pp. 36-53.

28. Osborne, M. J., And Rubinstein, A. A course in game theory. MIT press, 1994.

29. Shamir, A. How to share a secret. Communications of the ACM 22, 11 (1979), 612-613.

30. Yu, B., AND Singh, M. P. A social mechanism of reputation management in electronic communities. In 4th International Workshop on Cooperative Information Agents CIA (2000), vol. 1860 of LNCS, Springer, pp. 154-165. 